\title{
Validation of population receptive field estimates in human visual
}

\section{cortex}

\author{
Gurmukh Panesar ${ }^{1}$, Christina Moutsiana ${ }^{2}$ \& Dietrich Samuel Schwarzkopf $f^{3,4}$
}

1. School of Psychological Sciences, Birkbeck College, London, U.K.

2. Psychology, School of Social Sciences, University of Westminster, London, U.K.

3. Experimental Psychology \& Institute of Cognitive Neuroscience, University College London, U.K.

4. School of Optometry \& Vision Science, University of Auckland, New Zealand

* To whom all correspondence should be directed: c.moutsiana@westminster.ac.uk

Population receptive field (pRF) analysis has become a popular method for non-invasively inferring the spatial tuning properties of the human visual system and for reconstructing brain activity in visual space. Yet few studies have sought to validate pRF parameter estimates or systematically compared them between different methods. Here we used pRF models to reconstruct the visual cortex response to pseudo-randomly placed 'constellation' stimuli. We present different methods for visualizing brain activity in visual space. Compared to typically used back-projection of pRF profiles, we show that a searchlight approach using only pRF location greatly improves the spatial precision of reconstructions. We further quantify the precision with which different pRF estimates distinguish between stimulated and unstimulated parts of the visual field. Both combined wedge-and-ring and more conventional sweeping-bar stimuli afford excellent localization of the presented test stimuli. Even a probabilistic pRF model based on cortical anatomy without any retinotopic mapping data performs well albeit with lower precision than empirical data. Our findings demonstrate that pRF analysis is an accurate and robust method for mapping the position preference of voxels in human visual cortex.

\section{Introduction}

Neighboring neurons in retinotopic cortex respond selectively to neighboring locations of the visual field. Thus, functional magnetic resonance imaging ( $\mathrm{FMRI}$ ) can infer the location and size of the population receptive field of a voxel (Dumoulin and Wandell, 2008; Lee et al., 2013; Ress et al., 2011; Smith et al., 2001), each of which contains a neuronal population whose aggregate response also responds in a spatially selective way to a part of the visual field. In early brain areas, such as the primary visual cortex (V1), these pRFs tend to be small and densely packed. In higher extrastriate regions like the motion-sensitive medial temporal area 
or the object-sensitive lateral occipital complex, pRFs are much larger, sparser, and span much of the visual field (Amano et al., 2009; Dumoulin and Wandell, 2008).

PRF parameters are reliable across sessions (Benson et al., 2018; Senden et al., 2014; van Dijk et al., 2016) although attention can modulate pRF parameters (de Haas et al., 2014; Kay et al., 2015; Klein et al., 2014; Vo et al., 2017). PRF size differs in patients with amblyopia, achiasma, schizophrenia, and individuals with autism spectrum disorders (Anderson et al., 2016; Clavagnier et al., 2015; Hoffmann et al., 2012; Schwarzkopf et al., 2014). V1 pRF size correlates with perceptual biases for visual object size (Moutsiana et al., 2016) and with performance on various visual tasks (Song et al., 2015, 2013). Moreover, spatially selective pRFs have been demonstrated in face-sensitive fusiform cortex (Kay et al., 2015). Beyond the retinotopic domain, pRF methods have also been used to test the selectivity for the speed (Nishimoto et al., 2011), size (Harvey et al., 2015), and numerosity (Harvey et al., 2013) of visual stimuli, and for frequency preference of voxels in auditory cortex (Thomas et al., 2014).

Despite this research activity, there have been few confirmations of the accuracy of pRF parameters. First empirical evidence comes from experiments using them as an encoding model that reconstructs the stimulus observers were shown. Early experiments using this 'inverse retinotopy' approach could reproduce simple geometric patterns from cortical responses and even to reconstruct visual imagery (Thirion et al., 2006). Others used similar models to accurately identify the natural images or movies observers had viewed (Kay et al., 2008; Naselaris et al., 2015; Nishimoto et al., 2011; Zuiderbaan et al., 2017). More recently, similar approaches were used to project the contrast pattern of natural images back into visual space (Zipser, 2017). Another experiment used pRF modelling to analyze the relationship between orientation decoding performance and visual field location (Freeman et al., 2011). Critically, this analysis could only have worked if pRFs had accurately estimated the eccentricity of the stimuli.

Inverse retinotopy therefore represents an ideal means for validating the accuracy of $\mathrm{pRF}$ estimates. Here we reconstruct visual cortex activity produced by a set of stimuli pseudorandomly placed across the visual field (Figure 1A). This constitutes a proof-of-concept of how reconstruction approaches can be used to compare pRF methods. We use three different pRF mapping paradigms, two versions of a combined wedge-and-ring design and a sweeping bar design used in most pRF studies. We further use probabilistic predictions of pRF maps based on an atlas procedure (Benson et al., 2012). We quantify the precision with which these different pRF estimates distinguish between stimulated and unstimulated parts of the visual field. Moreover, we present a novel searchlight procedure for producing accurate reconstructions of cortical activity in visual space. 


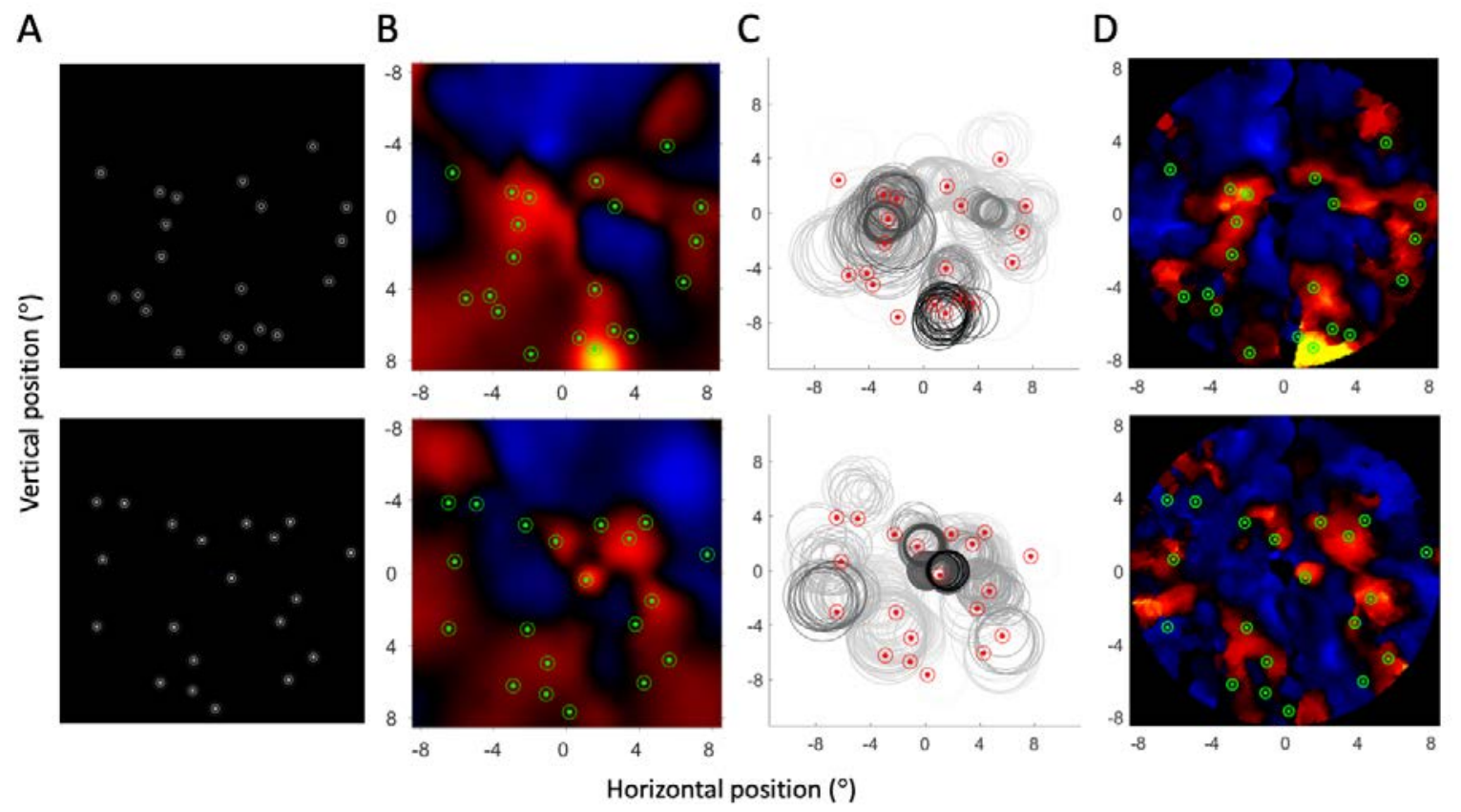

Figure 1. Different methods for projecting cortical activity back into visual space using V1 responses as an example. A. Two examples of 'constellation' stimuli a participant viewed. B. Standard back-projection approach multiplying the response to the stimulus in each voxel with its pRF profile and averaging across voxels. C. Scatter plot approach where each pRF is denoted by a circle indicating its pRF position and size, with the greyscale indicating the response to the constellation stimulus (red circles) in the $15 \%$ most responsive voxels (the darker, the stronger the response). D. Searchlight back-projection approach (see Materials and Methods). In B-D, the small green or red dots indicate the position of the 20 'star' stimuli. In B and D, red indicates positive response, blue indicates negative response relative to the mean.

\section{Materials and Methods}

\section{Participants}

Four healthy, right-handed, male observers (ages 30-39) with normal or corrected-to-normal vision participated in this experiment. All procedures were approved by the University College London Research Ethics Committee and participants gave written, informed consent.

\section{Procedure}

Participants took part in several fMRI scanning sessions. They participated in four sessions of retinotopic mapping, some of which were conducted for previous studies. Each session was conducted separately on different days, in some cases months or even years apart. In all experiments, participants were instructed to remain still and maintain fixation on a central dot throughout the experiment, and to press a button when a color change of the fixation dot 
occurred. In the mapping experiments using natural images as carrier stimuli, they were also asked to press a button when a target image appeared.

Moreover, participants were scanned in another session in which they viewed the 'constellation' stimuli (Figure 1A). In this experiment, they were asked to maintain fixation on a central green dot while 20 'stars' were presented in their visual field (see Constellation experiment below for details). The color of the fixation dot could turn red or blue to inform the participant when they broke fixation or when the eye tracking signal was lost. Participants were instructed to keep the dot green (maintain fixation). In addition, they were asked to interpret the stimuli they were presented with as constellations in the night sky and thus form perceptual groupings. For each of the five stimuli, they were asked to maintain the same groupings over the course of the experiment. Before the functional scans commenced, they were given one run of this experiment already in the bore to help them establish consistent groupings of their constellations. After scanning was completed, they viewed still images of the five stimuli and draw in the constellations they had formed using Microsoft Paint. This information was not used for any further analyses presented here.

\section{Constellation experiment}

In each scanning run, participants viewed five 'constellation' stimuli (Figure $1 \mathrm{~A}$ ) in a block design. Each participant was presented with a different set of constellation stimuli. The stimuli were generated as follows: Each constellation constituted an array of 20 white, pulsating circles on a black background. The location of the pulsating circles (the 'stars') were assigned pseudo-randomly, with the constraints that they must be within a circular region around fixation with the diameter of the screen height ( $17^{\circ}$ visual angle) and no star could be closer than $1^{\circ}$ from any of its neighbors.

Each star stimulus comprised 4 concentric grey rings (line thickness: $\sim 0.08^{\circ}$ ) whose diameters were initialized to range in equal steps from $0^{\circ}$ to approximately $1^{\circ}$. On each video frame, these diameters were increased/decreased by $0.006^{\circ}$, so that the rings where expanding/contracting. Whenever a ring diameter exceeded $1^{\circ}$ it was set to $0^{\circ}$; conversely, when it fell below $0^{\circ}$ it was set to $1^{\circ}$. Moreover, the luminance of the rings was proportional to their diameter so that it was maximal (white) at $0^{\circ}$ diameter and background (black) when it was $1^{\circ}$ diameter. This produced the impression that the center of the stimulus was bright but the rings faded with distance from the center. The direction of movement oscillated at $0.5 \mathrm{~Hz}$ : rings expanded in the first second of every stimulus presentation, then contracted in the next, and this repeated over the course of the stimulus block.

Constellation stimuli were presented for $12 \mathrm{~s}$ blocks, followed by a $6 \mathrm{~s}$ inter-trial interval containing only a black, blank screen. Each constellation stimulus was shown 3 times per run, and the 15 stimulus blocks were presented in a pseudo-randomized order. Each participant 
was scanned for 10 runs on this task. There were 280 volumes per run, including 10 dummy volumes at the beginning.

A fixation dot was constantly presented at the center of the screen. We used a magnetic resonance imaging compatible EyeLink 1000 eye tracking system to monitor the participants' eye movements throughout the experiment, sampled at $60 \mathrm{~Hz}$. Gaze contingent analysis of eye position ensured that participants maintained accurate fixation: If eye position remained within $1^{\circ}$ eccentricity of the fixation dot (diameter: $0.1^{\circ}$ ), the dot was green. If eye position strayed outside of this range, the dot turned red. If the eye tracking signal was lost due to blinks or other artifacts, the dot turned blue. Prior to the start of each run, participants viewed a blank screen with only the fixation dot for $10 \mathrm{~s}$. The dummy volumes were acquired during this time (see Scanning parameters below) and we used the median eye position during this period to calibrate our definition of the fixation position. A black annulus (width: $0.06^{\circ}$ ) surrounded the fixation dot at all times to ensure that the fixation dot was also visible if one of the star stimuli overlapped it.

\section{Sweeping-bar experiment}

We used sweeping bar apertures, similar to those used in previous studies (Dumoulin and Wandell, 2008; Schwarzkopf et al., 2014). Bars containing a ripple pattern (Alvarez et al., 2015; Schwarzkopf et al., 2014) were presented on a uniform grey background. To facilitate fixation compliance, a radar screen pattern consisting of 11 concentric circles and 12 radial lines dark grey lines extending from fixation was presented at all times. This radar screen pattern could extend beyond the edge of the screen.

Unlike in previous studies, the ripple pattern in this experiment was colored. To generate these colorful ripple stimuli, we generated the movie of ripples as previously (Alvarez et al., 2015; Schwarzkopf et al., 2014), but for the green color channel we offset the movie in time relative to the red color channel by $50 \%$, and in the blue color channel by $75 \%$.

The temporal order of the bar positions was the same across all runs. Bars were $\sim 0.95^{\circ}$ wide and were only visible within a circular region $17^{\circ}$ in diameter around fixation, so the maximal eccentricity of the bars was $8.5^{\circ}$. The bar traversed the screen in 1 second steps. Each step overlapped the previous bar position by one half of the bar width. There were 25 steps within each sweep and in each sweep the bar moved in one direction. The direction in each sweep were as follows: bottom-to-top, bottom-left-to-top-right, left-to-right, top-left-to-bottomright, top-to-bottom, top-right-to-bottom-left, right-to-left, bottom-right-to-top-left. After the fourth and the eighth sweep followed a blank period of 25 seconds during which only the fixation dot was displayed.

\section{Combined wedge+ring+localizer experiment}


We designed a stimulus combining rotating wedge and expanding/contracting ring apertures. There were 490 volumes in each run, including 10 dummy volumes at the beginning. Participants were scanned for three runs. Wedges and rings containing colorful images were presented against a uniform grey background. The same radar screen pattern as for the sweeping-bar experiment was presented at all times.

All combinations of wedge rotation (clockwise and counter-clockwise) and ring movement (expansion and contraction) were presented within the same run, interspersed with blank epochs. In addition to the wedge and ring cycles, the carrier stimuli inside the aperture alternated every 15 seconds between intact natural images and phase-scrambled versions of the same. The temporal order of wedge and ring positions was the same across all runs.

The position of the wedge and ring apertures updated every second. There were 60 positions of the wedge, and 36 positions of the ring per cycle. The maximal eccentricity of the stimuli was $8.5^{\circ}$. A small gap of $0.2^{\circ}$, tapered by a decreasing contrast ramp $\left(0.1^{\circ}\right.$ wide), surrounded the fixation dot $\left(0.13^{\circ}\right.$ diameter $)$. Wedges subtended a polar angle of $12^{\circ}$. The width of the rings was determined logarithmically as described previously (Moutsiana et al., 2016; van Dijk et al., 2016).

A mapping run was subdivided into four blocks lasting $90 \mathrm{~s}$ that were each followed by a blank period of $30 \mathrm{~s}$ during which only the fixation dot and radar screen pattern were presented. There were 1.5 cycles of the wedge rotation and 2.5 cycles of the ring expansion/contraction in each block. The direction of the apertures in each block was as follows: clockwise and expanding, clockwise and contracting, counter-clockwise and expanding, counter-clockwise and contracting. The carrier images began with intact images in the second and fourth block and with scrambled images in the first and second block.

The color of the fixation dot was usually black but every 0.2 seconds there was 0.03 probability that it could be changed for the next 0.2 seconds. Colors could be any random combination of maximum or minimum intensity in the red-green-blue color channels (including that it could remain black). If a color change had occurred, the fixation dot would revert back to black at least for the following 0.2 seconds. Participants were instructed to press a button whenever the dot turned red.

The carrier images were changed every 0.5 seconds in a pseudo-random order. The natural images were the same as in our previous studies (Moutsiana et al., 2016; van Dijk et al., 2016). This image set was produced by a Google Image search and depicted a wide range of different topics, from scenes of beaches, mountains, forests, and cityscapes, to faces, people, and writing in various scripts. In addition, one image was a tartan pattern (Anderson Modern), which appeared on average once or twice in a run. To direct their attention to the mapping 
stimuli, participants were instructed to press a button whenever this tartan pattern appeared, either as an intact image or as a phase-scrambled version. The images were always rotated along with the orientation of the wedge aperture so they would not be upright.

\section{Combined wedge+ring experiment}

We used another combined wedge-and-ring aperture design using natural images as a carrier but without phase-scrambled images. The design of these stimuli has already been described elsewhere (Moutsiana et al., 2016; van Dijk et al., 2016). In brief, we collected 12 runs of this design across two separate scanning sessions (usually several weeks apart). These two separate sessions were previously used to assess the test-retest reliability of pRF parameters (van Dijk et al., 2016) but for the analyses presented here the two sessions were averaged, that is, the same data as used in our previous study on size perception biases (Moutsiana et al., 2016). Two different directions (clockwise and expanding, anticlockwise and contracting) were presented in alternating runs. Each run contained 235 volumes, including the 10 dummy volumes at the beginning. There were 3 cycles of wedge rotation simultaneously presented with 5 cycles of ring expansion or contraction. The run concluded with a blank period of $45 \mathrm{~s}$. As in the other mapping experiments, the background was uniform grey but the radar screen pattern was presented at all times to facilitate fixation compliance.

\section{Scanning parameters}

We acquired magnetic resonance imaging data on a Siemens Avanto 1.5T scanner. For the combined wedge+ring stimuli and the constellation stimuli we used a 32-channel Siemens head coil with the front part removed, leaving 20 effective channels. For the sweeping-bars and combined wedge+ring+localizer stimuli we used a newer, customized 32-channel head coil in which the visor portion had been physically removed, leaving 30 effective channels. In either case, this ensured an unimpeded view of the stimulated part of the screen.

We acquired 36 transverse slices using a functional T2*-weighted multiband 2D echo-planar imaging pulse sequence (Breuer et al., 2005) with $2.3 \mathrm{~mm}$ isotropic voxels (field of view $96 \mathrm{x}$ 96, repetition time (TR) $1000 \mathrm{~ms}$, echo time (TE) $55 \mathrm{~ms}$, flip angle $75^{\circ}$, acceleration factor 4). Slices were oriented to be approximately centered on and parallel to the calcarine sulcus for each participant. The first ten volumes for each run in any experiment were removed to allow the signal to reach equilibrium. Finally, a T1-weighted anatomical magnetization-prepared rapid acquisition with gradient echo scan was acquired at a resolution of $1 \mathrm{~mm}$ isotropic (TR $2730 \mathrm{~ms}$, TE $3.57 \mathrm{~ms}$ ) during one of the two combined wedge-and-ring scanning sessions.

\section{Data preprocessing}


The $\mathrm{fMRI}$ data analysis including preprocessing and pRF analysis has already been described in detail (Alvarez et al., 2015; Moutsiana et al., 2016; Schwarzkopf et al., 2014; van Dijk et al., 2016). In short, the first 10 volumes were removed to allow the signal to reach equilibrium. Data were then preprocessed using SPM8 (Wellcome Trust Center for Neuroimaging; https://www.fil.ion.ucl.ac.uk/spm/software/spm8). Functional images were corrected for intensity inhomogeneities due to the head coil, and then realigned and unwarped to correct for head motion. Subsequently, they were coregistered to the structural image. We generated a three-dimensional model of the grey-white matter boundary of the cerebral cortex using FreeSurfer (http://surfer.nmr.mgh.harvard.edu/fswiki; version 5.3.0) and created inflated and spherical surfaces (Dale et al., 1999; Fischl et al., 1999).

Functional data were then further analyzed in MATLAB R2017a (Mathworks, Inc.) using SamSrf 5 (https://figshare.com/articles/SamSrf_toolbox_for_pRF_mapping/1344765), our custom toolbox for pRF mapping. All functional data were projected to the surface by finding the voxel at the middle position between the grey-white matter and the pial boundary for every vertex in the surface model. Separately for each scanning run, we applied linear detrending and z-standardization to the time series of every voxel project to the surface. For pRF mapping runs using the same temporal design (i.e., all runs in the combined wedge-andring-localizer and the sweeping bar designs, as well as even or odd runs in the combined wedge-and-ring design) we subsequently averaged the time series of individual runs.

\section{Analysis of pRF maps and constellation data}

We estimated the position and size of pRFs in occipital cortex using a forward modelling approach (Dumoulin and Wandell, 2008). We used a coarse-to-fine fitting approach described previously (Moutsiana et al., 2016; Schwarzkopf et al., 2014) where we first used an extensive grid search to grossly estimate the pRF parameters and then applied an optimization procedure to refine these parameters and also estimate the overall response magnitude.

The brain activity evoked by the constellation data were calculated by shifting the time series of each constellation run by 6 TRs to account for hemodynamic lag, and then averaging the responses of every voxel in visual cortex separately for the five constellation stimuli. This is essentially comparable to a general linear model using a simple boxcar regressor.

\section{Reconstructing cortical activity in visual space}

We used three different procedures to reconstruct visual cortex activity in visual space. The conventional procedure also used in several previous studies (Ekman et al., 2017; Kok et al., 2016a, 2016b; Kok and de Lange, 2014; Senden et al., 2019) multiplied the response measured to our constellation stimuli in a given voxel with a two-dimensional Gaussian profile of its pRF, and subsequently averaged these profiles for all voxels in a brain area. Because 
pRFs are considerably more clustered in some parts of the visual field (especially near the fovea because of cortical magnification), these average profiles were further normalized by dividing it by the overall frequency of pRFs, i.e. an average of all voxel pRF profiles where the activity of each voxel was set to 1 .

The second procedure was a simple scatter plot in which each pRF is depicted as a circle representing its position and size in the visual field. We chose the radius of the circle to be twice the standard deviation of the 2D Gaussian pRF (i.e. the approximate effective extent of the PRF) and the greyscale denoted the response to the 'constellation' stimuli. Such scatter plots are extremely cluttered when plotting all the pRFs in a region of interest. We therefore arbitrarily restricted these scatter plots to only $15 \%$ of pRFs that showed the strongest response to a given constellation stimulus. Moreover, we plotted pRFs in ascending order of their response strength, so that the pRF with the maximum response appeared on top of all others.

Finally, we also used a searchlight procedure for projecting cortical activity back into the visual field. Unlike the conventional procedure, this does not explicitly make use of pRF size but only pRF positions. A square of the visual field defined by the maximum eccentricity of the mapping stimulus $\left(8.5^{\circ}\right)$ was subdivided in a grid with steps of $0.1^{\circ}$. A circular searchlight with $0.5^{\circ}$-radius was then moved to each node of this grid and we quantified the mean response of all the pRFs whose centers fell inside the searchlight. The reconstruction then plots these average activity values at each of the grid nodes. Empty points were set to zero. While this method disregards pRF size explicitly, the size and density of pRFs is nevertheless reflected in the back-projection because more pRFs will be contained in foveal than peripheral searchlight locations.

\section{Quantifying the precision of stimulus responses}

We identified small regions of interest defined by the location of the 20 'star' stimuli in the visual field. All pRFs located within a $0.5^{\circ}$ radius of a star position were assigned to being part of the stars. The remainder were assigned to being part of the sky (i.e., the background). For each of the five constellations each participant viewed, we averaged the activity in the stars and the sky. For each participant, we then calculated a paired-t-test comparing the activity levels between stars and sky using the five constellations as individual observations. The tstatistic from this test thus quantifies how much larger the average response to stars is than to the sky, whilst taking into account the variance across the five constellations.

\section{Representational similarity analysis}

Finally, we also conducted a representational similarity analysis (Kriegeskorte et al., 2008) comparing the response patterns in a given visual region and the predicted response pattern 
using the stimulus locations and pRF parameters. Using our pRF models we predicted the activity produced by each of the constellation stimuli shown to a participant. We produced a stimulus mask, which was designed in the same way as the stimulus apertures masks used for pRF mapping: they were 100x100 pixel binary masks where background (sky) locations were set to 0 and the star locations set to 1 . We then used these stimulus masks to predict the response in each pRF by multiplying the Gaussian pRF profile with the stimulus mask and calculating the sum over all pixels in this convulsed image.

For each of the five constellation patterns shown to a given participant, we thus generated a vector of activity values for each of the pRFs in a brain region. As a measure of pattern similarity, we calculated the Pearson correlation between these predicted activity vectors and the actually observed activity vectors for the five constellations the participant had seen inside the scanner. Finally, we plotted the correlation matrix comparing the five predicted responses patterns to the five actually observed patterns. A very distinct encoding of the constellation stimuli would imply that there are high correlations along the diagonal of the correlation matrix, and low (near zero) correlations in off-diagonal cells. To quantify this, we calculated a two-sample t-test between the five diagonal correlations and the 20 off-diagonal cells.

\section{Results}

We used pRF parameters estimated by retinotopic mapping experiments to reconstruct the brain activity measured while participants viewed 'constellation' stimuli which each comprised 20 'star' stimuli (Figure 1A). First, we used a conventional approach for projecting brain activity back into visual space by multiplying the Gaussian pRF profile of each pRF with the measured $\mathrm{fMRI}$ response and averaging these across all the pRFs in a given brain area. This approach has been used in several previous studies (Ekman et al., 2017; Kok et al., 2016a, 2016b; Kok and de Lange, 2014) and can reconstruct some of the spatial locations of the presented stimuli (Figure 1B).

We also used scatter plots to project brain activity back into visual space (Figure 1C), which is similar to the back-projection approach used in other previous research (Kay et al., 2015). Since such scatter plots would be very cluttered when using all pRFs in a region of interest, we restricted these to only those pRFs showing the top $15 \%$ response to the constellation stimuli and plotting them in an order of ascending response. This again shows that pRFs close to the spatial location of presented stars respond to a given constellation stimulus.

Incorporating the spatial spread of pRFs averaging the pRF profiles tends to produce very blurred back-projections. This could underestimate the actual positional information contained in individual voxels. Conversely, the scatter plot approach shows that often large 
pRFs at relatively far distances from the stimuli respond. Unusually large pRFs could result from artefacts (such as movement or partial volume effects). Generally, due to the variability in pRF positions and density, the scatter plot approach may produce noisy and cluttered backprojections. It has also been shown that while the position estimates of pRF data have extremely high test-retest reliability, the reliability of pRF size estimates is considerably lower (Benson et al., 2018; van Dijk et al., 2016). The reason for this is probably that even very moderate variability will reduce the consistency of pRF sizes even if it does not affect the overall organization of visual field maps. PRF size is also a second-order measure compared to $\mathrm{pRF}$ position. Either way, greater variability in pRF size than pRF position implies that the former is likely to contribute more error to any reconstruction of the physical stimulus.
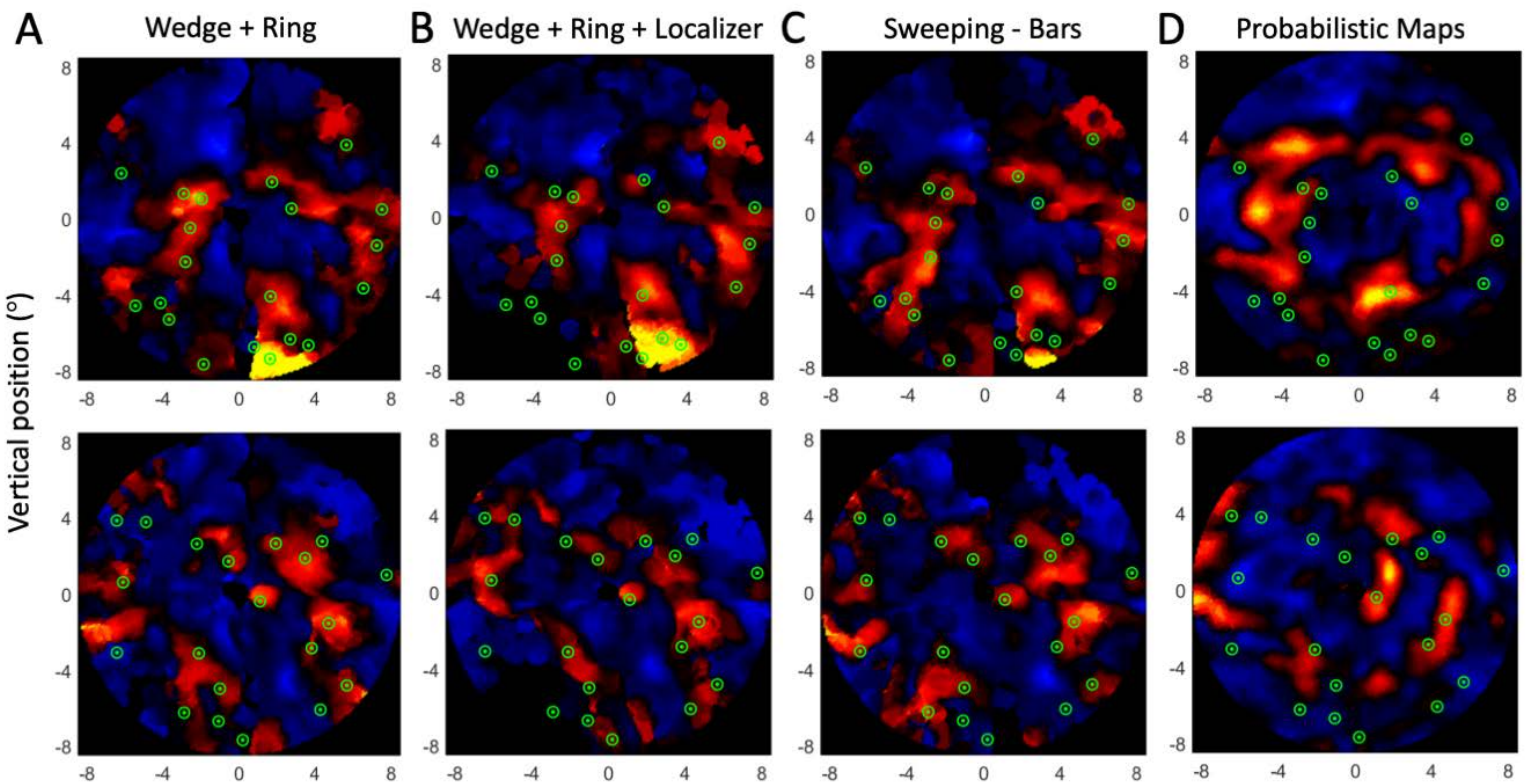

Horizontal position $\left({ }^{\circ}\right)$

Figure 2. Searchlight back-projections of V1 activity for two example constellation stimuli using four different pRF mapping designs. A. Combined wedgetring design (Moutsiana et al., 2016). B. Combined wedge+ring+localizer design. C. Conventional sweeping-bars design. D. Probabilistic map predictions (Benson et al., 2012). The small green dots indicate the position of the 20 'star' stimuli; red indicates positive response, blue indicates negative response relative to the mean.

We therefore designed another method for back-projecting brain activity in visual space. We employed a searchlight procedure, to quantify the response of pRFs within a confined region surrounding each visual field location. This does not use pRF size directly, however it still implicitly incorporates pRF sizes because the density pRFs decreases in the peripheral visual field as pRF size increases (Harvey and Dumoulin, 2011). The resulting back-projections provide considerably more spatial detail than the conventional approach. The activation corresponding to individual star stimuli is clearly distinguishable (Figure 1D). 
We next used this back-projection approach to visualize the brain response to the constellation stimuli using different methods for mapping pRFs. We used two mapping designs in which we combined wedge and ring apertures with natural images as carrier, one being data we already used in previous work (Moutsiana et al., 2016; van Dijk et al., 2016), the other an updated design that incorporated all directions of wedge rotation and ring movement in the same runs. In addition, we used a sweeping bar design (Dumoulin and Wandell, 2008) with a ripple carrier pattern similar to previous studies (Alvarez et al., 2015; Schwarzkopf et al., 2014). Finally, we used a probabilistic method based on a spatially normalized template employing the cortical folding pattern. This method predicts pRF positions in V1-V3 based on previous retinotopic mapping data in the normalized template (Benson et al., 2012).

The back-projections from all four methods accurately reflected the general spatial pattern of stimulus locations. The three empirical methods in which we had performed retinotopic mapping experiments produced reconstructions that corresponded very accurately to the actually presented stimuli (Figure $2 \mathrm{~A}-\mathrm{C}$ ). Reconstructions based on probabilistic maps were somewhat less precise, at least in some participants - however, they still produced recognizable reconstructions of the physical stimulus (Figure 2D).
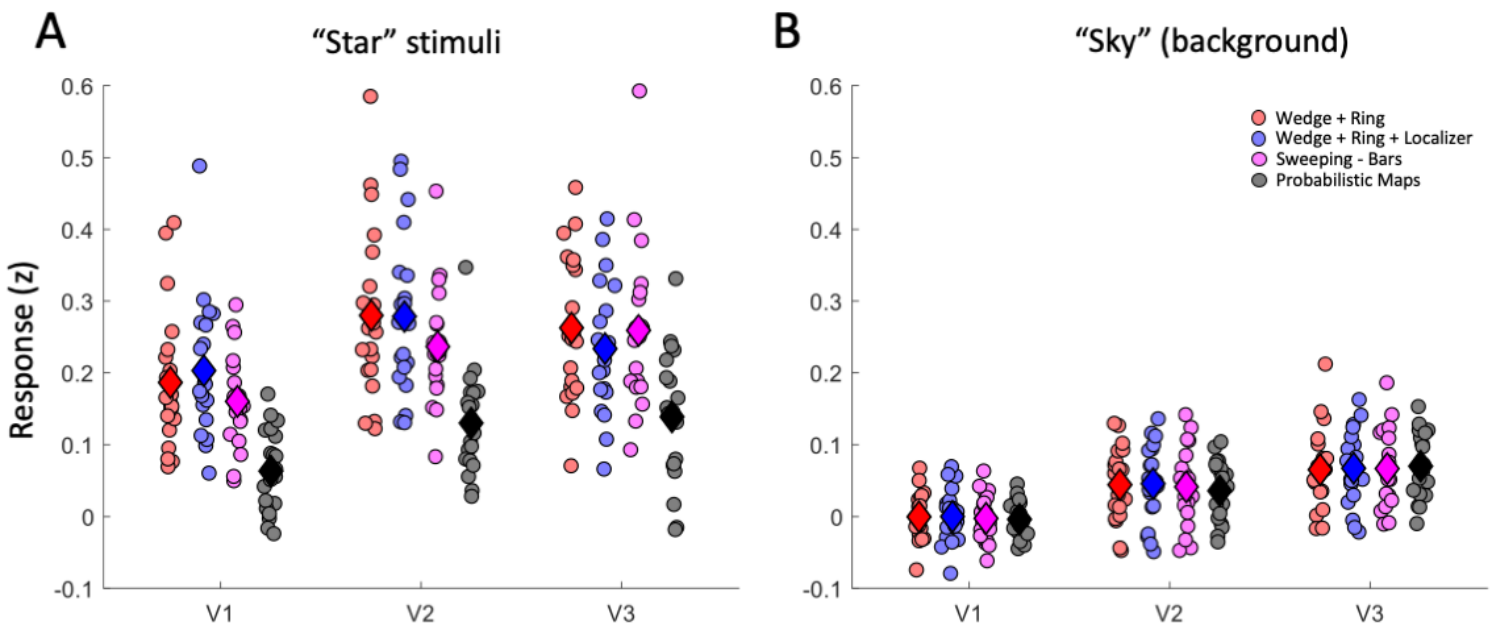

Figure 3. Responses to A. star stimuli and B. sky backgrounds. The response of V1-V3 pRFs in regions of interest defined by the actual positions of the star stimuli were averaged across each constellation stimulus and participant. Each dot denotes the average response to a stimulus and participant. The diamond symbols show the group means.

\section{The precision of $p R F$ models}

Thus far, our comparison of these back-projections has been solely based on visual inspection. We therefore quantified the precision of the pRF-based reconstruction by determining how accurately they could distinguish between visual field locations containing 'star' stimuli 
compared to the 'sky' background. For each of the five constellation stimuli viewed by a participant, we determined all the pRFs that fell within $0.5^{\circ}$ of any star stimulus. We then calculated the mean response of these pRFs and the mean response across all the remaining pRFs in the unstimulated 'sky' background (restricted to the mapped visual field up to an eccentricity of $8.5^{\circ}$ ).

The average responses in different visual areas to stars and sky for each of the five constellations and all four participants are shown in Figure 3. For all pRF methods used, the responses to 'star' stimuli were considerably greater than to the 'sky' background. The responses to stars were also very similar for all three retinotopic mapping procedures (Figure $3 \mathrm{~A})$. The response as estimated by the probabilistic map procedure was lower but still well above the level of the sky background region (Figure 3B). In addition, the response to the sky region also increased steadily from V1, V2 and V3, while the response to stars seemed more constant, although it was somewhat lower in V1 than in the extrastriate cortex.

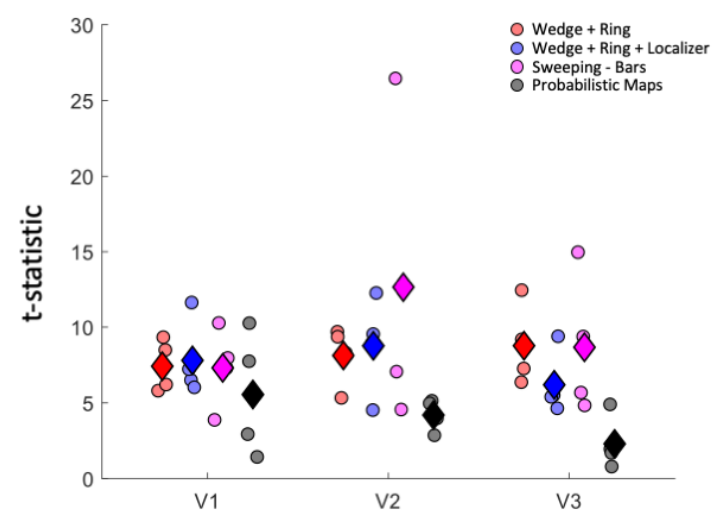

Figure 4. Precision of stimulus reconstructions. The t-statistics on a paired t-test comparing the mean of star and sky responses in each participant (treating the five constellations per participant as individual observations) are plotted for each visual area for the different pRF mapping designs. Dots denote individual participants. Diamond symbols denote the group means.

To further estimate the precision of these reconstructions, we calculated a paired t-test for each participant comparing the mean star and sky responses and treating the five constellation stimuli as individual observations. The t-statistic from this test essentially quantifies how discriminable the response to stars is from the response to skies, given the variability across the five stimuli. Figure 4 shows the mean t-statistic across participants for the different visual areas. For all three retinotopic mapping methods and across all visual areas the t-statistic was comparably high (but note the outlier for V2). In contrast, for the probabilistic map method t-statistics were considerably lower, especially in V2 and V3.

Finally, we also performed a representational similarity analysis (Kriegeskorte et al., 2008). We used the pRF parameters to predict the response to each of the five constellations presented to a given participant and correlated these predicted response patterns with the 
actually observed responses. Figure 5 shows the correlation matrices for V1 in two example participants for the four pRF mapping designs. For all participants and mapping conditions, the diagonal correlations were stronger than the off-diagonal cells. This indicates that the response patterns predicted based on each stimulus were a better match with the actually observed response patterns than the other stimuli.

We further quantified these results by averaging the correlations in the diagonal cells (Figure $6 \mathrm{~A})$. This revealed a tendency for both combined wedge and ring mapping designs to produce somewhat stronger correlations than either the sweeping-bars design or the probabilistic mapping method. Predictions using the sweeping bars design were also substantially lower than those of the other methods in V1, a result that was very consistent across the four participants.
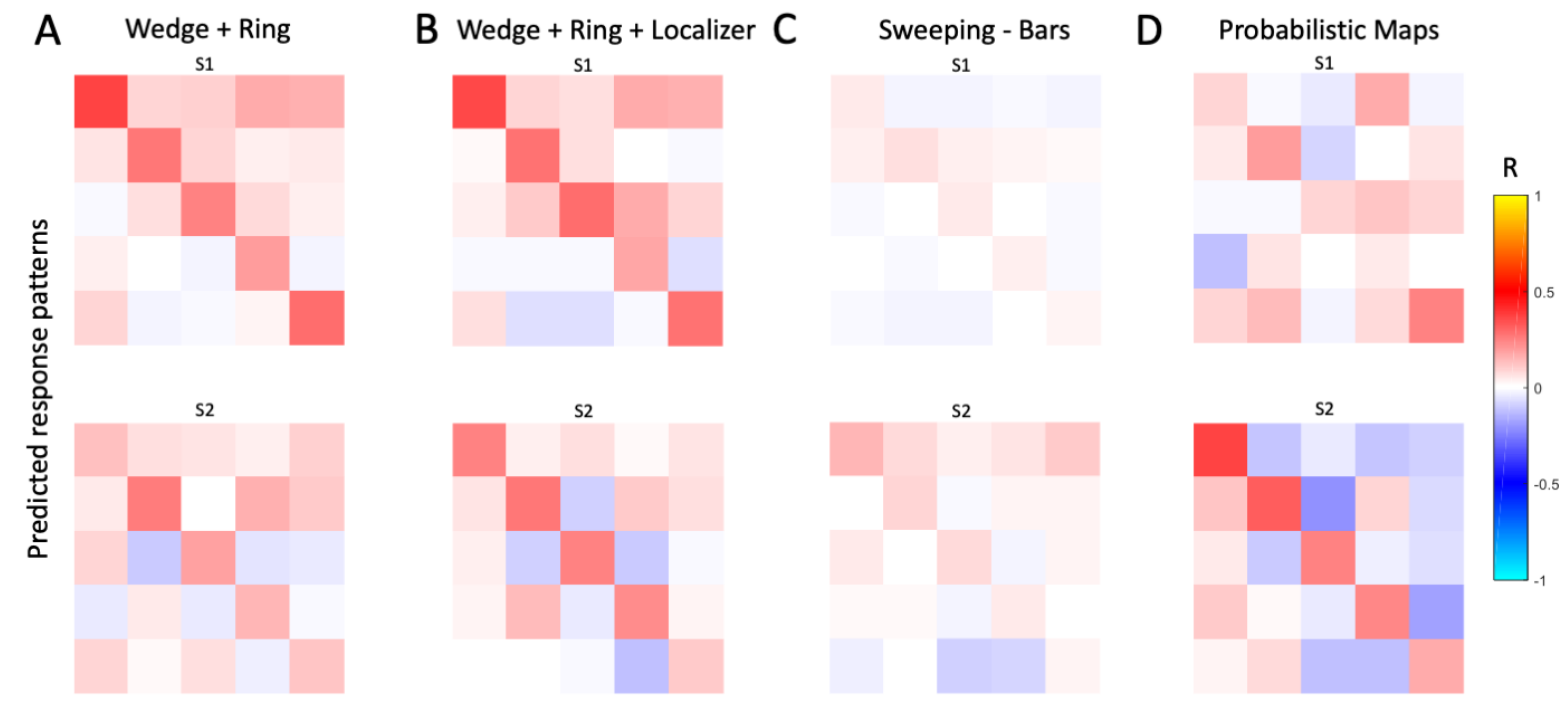

Observed response patterns

Figure 5. Representational similarity analysis. Correlation matrices from two participants (rows) showing the pattern similarity between observed responses across pRFs in V1 and the pRF-based predictions using $A$. the combined wedgetring design (Moutsiana et al., 2016), B. the combined wedge+ring+localizer design, C. the sweeping-bars design, or D. the probabilistic mapping method (Benson et al., 2012). Hot colors denote positive correlations, cold colors negative correlations. Each row and column of the matrix refers to one of the five constellation stimuli.

To determine the confusability between the predictions from the different constellation stimuli, we again calculated a t-statistic, this time comparing the correlations in diagonal cells to those in off-diagonal cells (Figure 6B). This again showed greater precision, reflected by larger $\mathrm{t}$-statistics, for the combined wedge and ring mapping designs than the sweeping-bars and probabilistic mapping method. Interestingly, this completely cancelled out the generally lower correlations for the sweeping-bars design especially in V1: the t-statistics for sweepingbars were largely comparable to those of the probabilistic mapping method. 
A

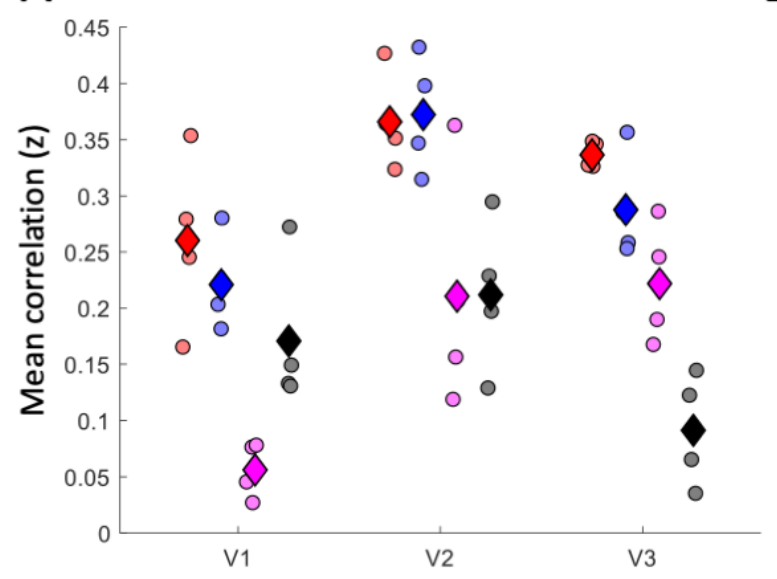

B

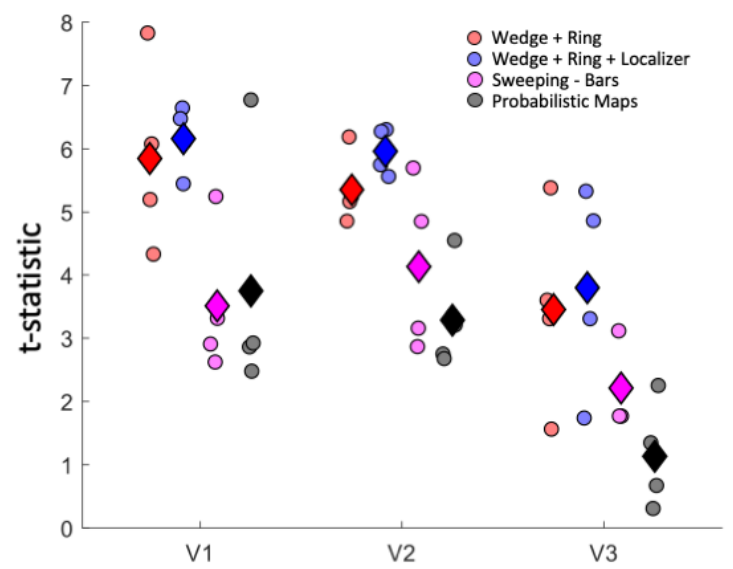

Figure 6. Quantification of representational similarity matrices in V1-V3 for the different pRF mapping methods. A. Mean correlation of diagonal cells. B. Confusability index calculated as the t-statistic on a t-test between correlations for diagonal and off-diagonal cells. Dots denote data from individual participants. Diamond symbols denote the group means.

\section{Discussion}

We show that three different stimulation paradigms for pRF mapping all produce robust estimates of the spatial selectivity of visual cortex. A probabilistic procedure for estimating pRFs based on an atlas of previous data is also effective, albeit with somewhat reduced precision.

An early study conducted simulations to argue that the estimates of pRF eccentricity (the distance from the center of gaze) are more accurate than when using traditional procedures based on the travelling-wave principle (Dumoulin and Wandell, 2008). Another study confirmed the theoretical prediction that eccentricity estimates from travelling-wave analysis are skewed relative to those obtained with pRF modelling (Alvarez et al., 2015). However, both of these comparisons tacitly work on the theoretical assumption that estimates derived from pRF modelling are in fact accurate. Our present data confirm this assumption: when the exact location of the stimuli in the visual field is known, an accurate pRF model can reconstruct stimulus locations.

The current study is not the first to use this kind of pRF model to predict or reconstruct visual stimuli. Previous studies used comparable models to visualize the brain activity in response to natural images (Zipser, 2017) while others used them to decode stimulus identity using a very similar correlation-based algorithm to the one we employed here (Zuiderbaan et al., 2017). We could therefore be confident that this approach to reverse retinotopy (Thirion et 
al., 2006) was feasible. Our aim here was to test if various experimental designs for obtaining pRF maps differed in the precision with which they estimated the visual field location preferences of voxels.

Our findings suggest that pRF maps obtained with the three empirical experimental designs are highly comparable and accurately estimate visual field position. By and large, the two designs based on a stimulus that combines rotating wedges and expanding/contracting rings seemed to outperform the more traditional sweeping bar design. This mirrors the results from our previous study in which wedge and ring stimuli yielded greater goodness-of-fit of the pRF model than did sweeping bar stimuli (Alvarez et al., 2015). However, those previous results had the caveat that the model fits of different stimulus designs are not statistically comparable. We speculate that the reason for poorer model fits for bars result from the difference in duty cycle: In the wedge and ring design more time is dedicated to stimulating the visual field and there are more repetitions of the same condition. The design is also more efficient because it simultaneously modulates the polar angle and eccentricity of the stimulus while the bar stimulus only varies along one axis at a time. One study recently employed a more efficient bars design combining bars of orthogonal orientations (Miranda et al., 2018). The approach we present here could be used to test if such an efficient design improves the precision of the pRF maps compared to a single bar design.

It is important to note that unlike in our previous work (Alvarez et al., 2015), we did not set out to match characteristics of these different mapping designs in the present study. The two combined wedge and ring designs differed from one another and from the sweeping-bars design in terms of the amount of data that was collected and how many images were acquired per scanning run. Naturally, with only four participants these analyses would also not have sufficient statistical power to detect subtle differences in the precision of these designs. We therefore do not make any strong claims about the relative differences between the designs. Some of the differences seem consistent across participants and the various analyses. In particular, our results indicate that the precision of the probabilistic maps method is poorer than those of the empirical mapping experiments. This is unsurprising given it is only a statistical prediction derived on the anatomical features using pRF mapping data acquired in a different study under very different conditions (Benson et al., 2012). Recently, it was shown that a Bayesian approach for fitting an atlas map to limited mapping data can improve the quality of these predicted maps substantially (Benson and Winawer, 2018). Future studies could use the approach we present here to test the accuracy of these predicted Bayesian maps.

While our findings cannot permit any comprehensive inferences about the best pRF design, our study illustrates the concept of reconstructing visual stimuli to estimate the accuracy of pRF methods. By testing how accurately responses to stimulated locations can be distinguished from the unstimulated background, we can test the precision of the underlying 
pRF map relative to a known ground truth, the true location of the stimuli in visual space. This already demonstrates some basic patterns that are highly consistent even across our small sample, such as the increase in responses to the background as we move up the visual hierarchy. Presumably this is because pRF sizes increase and thus the distinction between stimulus and background becomes more blurred.

\section{Back-projection of visual cortical activity}

We also compared three different methods for using pRF maps to project visual cortical responses back into the visual space. Such visualizations are becoming increasingly popular and have been used to investigate the topographic response patterns to illusory contours (Kok et al., 2016a; Kok and de Lange, 2014), expectation effects (Ekman et al., 2017; Kok et al., 2016b), and attentional processing (Kay et al., 2015; Vo et al., 2017). Similar approaches have also been used to reconstruct the visual stimuli presented to participants and even the contents of visual imagery (Senden et al., 2019; Thirion et al., 2006). The typical approach to this averages the profiles of individual pRFs multiplied by the activity in their respective voxel. This results in very blurry images as pRFs can subtend considerable amounts of visual space.

Another approach is to plot back pRFs denoted by a circle in a scatter graph. This has for example been used to study the effect of selective attention in face-processing areas in the ventral stream (Kay et al., 2015). Back-projections using this approach are less blurry than when averaging pRF profiles, but they appear cluttered and it can be difficult to visualize the response magnitude, especially when the sign is also relevant.

Instead, we present here a third approach based on a searchlight algorithm. Here we sample pRFs whose centers fall within a small searchlight region which is sequentially moved throughout the visual field. Activity values at each searchlight position are then visualized as a pixel in an image. This approach does not directly incorporate pRF size. It does however implicitly use pRF size because larger pRFs in more peripheral visual field locations also tend to be less dense (Harvey and Dumoulin, 2011). We found that this searchlight approach produces much more well-defined back-projections than those based on the conventional approaches. While the general stimulus structure was apparent in all back-projections, the searchlight algorithm contained more focal activation spots that largely coincide with the position of the visual stimuli. Thus, using less information, specifically pRF size, can actually improve the inferences that can be drawn from these data. The fact that pRF size information can hamper our interpretation of the results is in keeping with the finding that pRF size estimates are less reliable than pRF position estimates (Benson et al., 2018; van Dijk et al., 2016).

\section{Conclusions}


Our findings demonstrate that pRF mapping can accurately measure the spatial selectivity of voxels in visual cortex. Irrespective of the stimulation paradigm used, pRF parameter estimates, and the resulting reconstruction of visual activity produced by an independent experiment, were highly similar for all our designs. Combined stimuli simultaneously presenting rotating wedges and expanding/contracting rings produced just as precise pRF data as the more conventional design using sweeping bars. Combined stimulus designs are arguably more efficient and especially the use of a ring stimulus might facilitate fixation compliance because they are more symmetric around fixation. Finally, we also presented a novel searchlight procedure for projecting cortical activity back into visual space. This shows that spatially highly accurate information can be reconstructed from visual cortical activity beyond that which has been shown with previous approaches.

\section{Acknowledgements}

This work was supported by a ERC Starting Grant to DSS. We thank Susanne Stoll for useful discussions.

\section{Data availability}

Processed data necessary for reproducing the main results, analysis functions, and stimulus presentation scripts are available for download at osf.io/4c5ax

\section{References}

Alvarez, I., De Haas, B.A., Clark, C.A., Rees, G., Schwarzkopf, D.S., 2015. Comparing different stimulus configurations for population receptive field mapping in human fMRI. Front. Hum. Neurosci 9, 96. https://doi.org/10.3389/fnhum.2015.00096

Amano, K., Wandell, B.A., Dumoulin, S.O., 2009. Visual field maps, population receptive field sizes, and visual field coverage in the human MT+ complex. J. Neurophysiol 102, 2704-2718. https://doi.org/10.1152/jn.00102.2009

Anderson, E.J., Tibber, M.S., Schwarzkopf, D.S., Shergill, S.S., Fernandez-Egea, E., Rees, G., Dakin, S.C., 2016. Visual population receptive fields in people with schizophrenia have reduced inhibitory surrounds. J. Neurosci. https://doi.org/10.1523/JNEUROSCI.3620-15.2016

Benson, N.C., Butt, O.H., Datta, R., Radoeva, P.D., Brainard, D.H., Aguirre, G.K., 2012. The Retinotopic Organization of Striate Cortex Is Well Predicted by Surface Topology. Curr. Biol. https://doi.org/10.1016/j.cub.2012.09.014

Benson, N.C., Jamison, K.W., Arcaro, M.J., Vu, A.T., Glasser, M.F., Coalson, T.S., Van Essen, D.C., Yacoub, E., Ugurbil, K., Winawer, J., Kay, K., 2018. The Human Connectome 
Project 7 Tesla retinotopy dataset: Description and population receptive field analysis. J Vis 18, 23. https://doi.org/10.1167/18.13.23

Benson, N.C., Winawer, J., 2018. Bayesian analysis of retinotopic maps. Elife 7. https://doi.org/10.7554/eLife.40224

Breuer, F.A., Blaimer, M., Heidemann, R.M., Mueller, M.F., Griswold, M.A., Jakob, P.M., 2005. Controlled aliasing in parallel imaging results in higher acceleration (CAIPIRINHA) for multi-slice imaging. Magn Reson Med 53, 684-691. https://doi.org/10.1002/mrm.20401

Clavagnier, S., Dumoulin, S.O., Hess, R.F., 2015. Is the Cortical Deficit in Amblyopia Due to Reduced Cortical Magnification, Loss of Neural Resolution, or Neural Disorganization? J. Neurosci. 35, 14740-14755. https://doi.org/10.1523/JNEUROSCI.1101-15.2015

Dale, A.M., Fischl, B., Sereno, M.I., 1999. Cortical surface-based analysis. I. Segmentation and surface reconstruction. Neuroimage 9, 179-194. https://doi.org/10.1006/nimg.1998.0395

de Haas, B., Schwarzkopf, D.S., Anderson, E.J., Rees, G., 2014. Perceptual load affects spatial tuning of neuronal populations in human early visual cortex. Curr. Biol. 24, R66-67. https://doi.org/10.1016/j.cub.2013.11.061

Dumoulin, S.O., Wandell, B.A., 2008. Population receptive field estimates in human visual cortex. Neuroimage 39, 647-660. https://doi.org/10.1016/j.neuroimage.2007.09.034

Ekman, M., Kok, P., de Lange, F.P., 2017. Time-compressed preplay of anticipated events in human primary visual cortex. Nat Commun 8, 15276. https://doi.org/10.1038/ncomms15276

Fischl, B., Sereno, M.I., Dale, A.M., 1999. Cortical surface-based analysis. II: Inflation, flattening, and a surface-based coordinate system. Neuroimage 9, 195-207. https://doi.org/10.1006/nimg.1998.0396

Freeman, J., Brouwer, G.J., Heeger, D.J., Merriam, E.P., 2011. Orientation decoding depends on maps, not columns. J. Neurosci 31, 4792-4804. https://doi.org/10.1523/JNEUROSCI.5160-10.2011

Harvey, B.M., Dumoulin, S.O., 2011. The Relationship between Cortical Magnification Factor and Population Receptive Field Size in Human Visual Cortex: Constancies in Cortical Architecture. J. Neurosci. 31, 13604-13612. https://doi.org/10.1523/JNEUROSCI.2572-11.2011

Harvey, B.M., Fracasso, A., Petridou, N., Dumoulin, S.O., 2015. Topographic representations of object size and relationships with numerosity reveal generalized quantity processing in human parietal cortex. Proc. Natl. Acad. Sci. U.S.A. 112, 13525-13530. https://doi.org/10.1073/pnas.1515414112

Harvey, B.M., Klein, B.P., Petridou, N., Dumoulin, S.O., 2013. Topographic representation of numerosity in the human parietal cortex. Science 341, 1123-1126. https://doi.org/10.1126/science.1239052

Hoffmann, M.B., Kaule, F.R., Levin, N., Masuda, Y., Kumar, A., Gottlob, I., Horiguchi, H., Dougherty, R.F., Stadler, J., Wolynski, B., Speck, O., Kanowski, M., Liao, Y.J., Wandell, B.A., Dumoulin, S.O., 2012. Plasticity and stability of the visual system in human achiasma. Neuron 75, 393-401. https://doi.org/10.1016/j.neuron.2012.05.026

Kay, K.N., Naselaris, T., Prenger, R.J., Gallant, J.L., 2008. Identifying natural images from human brain activity. Nature 452, 352-355. https://doi.org/10.1038/nature06713 
Kay, K.N., Weiner, K.S., Grill-Spector, K., 2015. Attention reduces spatial uncertainty in human ventral temporal cortex. Curr. Biol. 25, 595-600.

https://doi.org/10.1016/j.cub.2014.12.050

Klein, B.P., Harvey, B.M., Dumoulin, S.O., 2014. Attraction of Position Preference by Spatial Attention throughout Human Visual Cortex. Neuron. https://doi.org/10.1016/j.neuron.2014.08.047

Kok, P., Bains, L.J., van Mourik, T., Norris, D.G., de Lange, F.P., 2016a. Selective Activation of the Deep Layers of the Human Primary Visual Cortex by Top-Down Feedback. Curr. Biol. 26, 371-376. https://doi.org/10.1016/j.cub.2015.12.038

Kok, P., de Lange, F.P., 2014. Shape perception simultaneously up- and downregulates neural activity in the primary visual cortex. Curr. Biol. 24, 1531-1535. https://doi.org/10.1016/j.cub.2014.05.042

Kok, P., van Lieshout, L.L.F., de Lange, F.P., 2016b. Local expectation violations result in global activity gain in primary visual cortex. Sci Rep 6, 37706. https://doi.org/10.1038/srep37706

Kriegeskorte, N., Mur, M., Bandettini, P., 2008. Representational similarity analysis connecting the branches of systems neuroscience. Front Syst Neurosci 2, 4. https://doi.org/10.3389/neuro.06.004.2008

Lee, S., Papanikolaou, A., Logothetis, N.K., Smirnakis, S.M., Keliris, G.A., 2013. A new method for estimating population receptive field topography in visual cortex. Neuroimage 81, 144-157. https://doi.org/10.1016/j.neuroimage.2013.05.026

Miranda, Â.S.C., Rosa, A. de F.M., Dias, M.J.P., Harvey, B.M., Silva, M.F.L. da, Castelo-Branco, M. de S. e S., Murta, J.C.N., 2018. Optical Properties Influence Visual Cortical Functional Resolution After Cataract Surgery and Both Dissociate From Subjectively Perceived Quality of Vision. Invest. Ophthalmol. Vis. Sci. 59, 986-994. https://doi.org/10.1167/iovs.17-22321

Moutsiana, C., de Haas, B., Papageorgiou, A., van Dijk, J.A., Balraj, A., Greenwood, J.A., Schwarzkopf, D.S., 2016. Cortical idiosyncrasies predict the perception of object size. Nat Commun 7, 12110. https://doi.org/10.1038/ncomms12110

Naselaris, T., Olman, C.A., Stansbury, D.E., Ugurbil, K., Gallant, J.L., 2015. A voxel-wise encoding model for early visual areas decodes mental images of remembered scenes. Neuroimage 105, 215-228.

https://doi.org/10.1016/j.neuroimage.2014.10.018

Nishimoto, S., Vu, A.T., Naselaris, T., Benjamini, Y., Yu, B., Gallant, J.L., 2011. Reconstructing visual experiences from brain activity evoked by natural movies. Curr. Biol. 21, 16411646. https://doi.org/10.1016/j.cub.2011.08.031

Ress, D., Greene, C., Dumoulin, S.O., Harvey, B., 2011. Tomographic measurement of population receptive fields in early visual cortex. J Vis 11, 1197-1197. https://doi.org/10.1167/11.11.1197

Schwarzkopf, D.S., Anderson, E.J., Haas, B. de, White, S.J., Rees, G., 2014. Larger Extrastriate Population Receptive Fields in Autism Spectrum Disorders. J. Neurosci. 34, 27132724. https://doi.org/10.1523/JNEUROSCI.4416-13.2014

Senden, M., Emmerling, T.C., van Hoof, R., Frost, M.A., Goebel, R., 2019. Reconstructing imagined letters from early visual cortex reveals tight topographic correspondence between visual mental imagery and perception. Brain Struct Funct. https://doi.org/10.1007/s00429-019-01828-6 
Senden, M., Reithler, J., Gijsen, S., Goebel, R., 2014. Evaluating Population Receptive Field Estimation Frameworks in Terms of Robustness and Reproducibility. PLOS ONE 9, e114054. https://doi.org/10.1371/journal.pone.0114054

Smith, A.T., Singh, K.D., Williams, A.L., Greenlee, M.W., 2001. Estimating receptive field size from $\mathrm{fMRI}$ data in human striate and extrastriate visual cortex. Cereb. Cortex 11, 1182-1190.

Song, C., Schwarzkopf, D.S., Kanai, R., Rees, G., 2015. Neural Population Tuning Links Visual Cortical Anatomy to Human Visual Perception. Neuron 85, 641-56. https://doi.org/10.1016/j.neuron.2014.12.041

Song, C., Schwarzkopf, D.S., Rees, G., 2013. Variability in visual cortex size reflects tradeoff between local orientation sensitivity and global orientation modulation. Nat Commun 4, 2201. https://doi.org/10.1038/ncomms3201

Thirion, B., Duchesnay, E., Hubbard, E., Dubois, J., Poline, J.-B., Lebihan, D., Dehaene, S., 2006. Inverse retinotopy: inferring the visual content of images from brain activation patterns. Neuroimage 33, 1104-1116.

https://doi.org/10.1016/j.neuroimage.2006.06.062

Thomas, J.M., Huber, E., Stecker, G.C., Boynton, G.M., Saenz, M., Fine, I., 2014. Population receptive field estimates of human auditory cortex. Neurolmage. https://doi.org/10.1016/j.neuroimage.2014.10.060

van Dijk, J.A., de Haas, B., Moutsiana, C., Schwarzkopf, D.S., 2016. Intersession reliability of population receptive field estimates. Neuroimage. https://doi.org/10.1016/j.neuroimage.2016.09.013

Vo, V.A., Sprague, T.C., Serences, J.T., 2017. Spatial Tuning Shifts Increase the Discriminability and Fidelity of Population Codes in Visual Cortex. J. Neurosci. 37, 3386-3401. https://doi.org/10.1523/JNEUROSCI.3484-16.2017

Zipser, K., 2017. Visualizing fMRI BOLD responses to diverse naturalistic scenes using retinotopic projection. Journal of Vision 17, 18-18. https://doi.org/10.1167/17.6.18

Zuiderbaan, W., Harvey, B.M., Dumoulin, S.O., 2017. Image identification from brain activity using the population receptive field model. PLoS ONE 12, e0183295. https://doi.org/10.1371/journal.pone.0183295 